\title{
Increasing the Equilibrium Solubility of Dopants in Semiconductor Multilayers and Alloys
}

\author{
A. Höglund, ${ }^{1}$ O. Eriksson, ${ }^{1}$ C. W. M Castleton, ${ }^{2}$ and S. Mirbt ${ }^{1}$ \\ ${ }^{1}$ Condensed Matter Theory Group, Department of Physics, Uppsala University, Box 530, SE-751 21 Uppsala, Sweden \\ ${ }^{2}$ Department of Materials Chemistry, Uppsala University, Box 538, SE-751 21, Uppsala, Sweden
}

(Received 29 March 2007; published 11 March 2008)

\begin{abstract}
We have theoretically studied the possibility to control the equilibrium solubility of dopants in semiconductor alloys, by strategic tuning of the alloy concentration. From the modeled cases of $\mathrm{C}^{0}$ in $\mathrm{Si}_{x} \mathrm{Ge}_{1-x}, \mathrm{Zn}^{-}$and $\mathrm{Cd}^{-}$in $\mathrm{Ga}_{x} \mathrm{In}_{1-x} \mathrm{P}$ it is seen that under certain conditions the dopant solubility can be orders of magnitude higher in an alloy or multilayer than in either of the elements of the alloy. This is found to be due to the solubility's strong dependence on the lattice constant for size mismatched dopants. The equilibrium doping concentration in alloys or multilayers could therefore be increased significantly. More specifically, $\mathrm{Zn}^{-}$in a $\mathrm{Ga}_{x} \mathrm{In}_{1-x} \mathrm{P}$ multilayer is found to have a maximum solubility for $x=0.9$, which is 5 orders of magnitude larger than that of pure InP.
\end{abstract}

DOI: 10.1103/PhysRevLett.100.105501

Attaining significantly higher equilibrium doping concentrations is of great importance for the development of future semiconductor technologies. In the nonequilibrium case, raised doping concentrations $\sim 10^{20} \mathrm{~cm}^{-3}$ have been achieved by epitaxial methods in $\mathrm{Si}_{1-x} \mathrm{Ge}_{x}: \mathrm{B}$ [1-3], GaAs:N [4], GaInAs:Be [5], GaAs:C [6], and C in diamond [7]. Even higher, still electrically active, doping concentrations of up to $10^{22} \mathrm{~cm}^{-3}$ have been achieved in localized layers by so called $\delta$-doping [8-10]. From a theoretical point of view the raised nonequilibrium concentrations have been successfully explained by the increased range of the chemical potential of the dopant during epitaxial growth [11-13].

In this Letter we consider ways to increase the solid solubility to achieve higher doping concentrations under equilibrium conditions. We study the dependence of the equilibrium solubility of substitutional defects, including dopants, in compound semiconductors $A_{x} B_{1-x}$. We demonstrate that the equilibrium solubility of dopants need not be monotonic in $x$ and lie between those of the pure materials $A$ and $B$. Instead, we will show that careful tuning of $x$ can result in equilibrium solubilities many orders of magnitude higher in the alloy than in either of the pure materials.

It is well known that high doping can alter the lattice constant of a material [14]. Here we investigate the possibility of using changes in the lattice constant to control the doping concentration. Initial indications of this have been noted in previous experimental work [15], in theoretical studies of dopants in silicon under applied biaxial strain [16] and for vacancies and self-interstitials using a phenomenological method [17]. However, the full implications of this possibility have not so far been realized.

Our hypothesis builds on the way in which the solubility depends on the difference between the dopant's and the substituted host atom's equilibrium bond lengths. The solubility of a dopant atom of the same size and equilibrium bonding distance as the host atoms will have little
PACS numbers: 61.72.uf, 61.66.Dk, 61.72.uj, 71.15.Mb

dependence on the strain of the material since the difference in bond energy will be constant. If the equilibrium bonding distances differ, however, the solubility of the dopant may have a strong dependence on the lattice constant, such that the equilibrium solubility of smaller (larger) dopants increases under compressive (expansive) strain. Our results show for the first time that the equilibrium concentration of a defect can sometimes be orders of magnitude higher in an alloy or multilayer than in either of the constituents. This leads to the possibility of increasing the equilibrium solubilities of dopants through strategically composing multilayers or alloys.

The equilibrium solubility may be predicted through the calculation of the defect formation energy $\varepsilon^{\text {form }}[18]$

$$
C=N_{s} e^{-\varepsilon^{\text {form }} / k_{B} T},
$$

where $N_{s}$ is the concentration of possible lattice sites for the defect. Our calculations are performed using plane wave $a b$ initio density functional theory [19] within the local density approximation (LDA) together with ultrasoft pseudopotentials [20]. A full description of technical details and more extensive results are presented elsewhere [21] (the small formation entropy contribution is expected to have a negligible dependence on strain).

The $\mathrm{C}_{\mathrm{IV}}^{0}$ impurity in $\mathrm{Si}_{x} \mathrm{Ge}_{1-x}$ and the $\mathrm{Zn}_{\text {III }}^{-}$and $\mathrm{Cd}_{\text {III }}^{-}$ dopants in $\mathrm{Ga}_{x} \mathrm{In}_{1-x} \mathrm{P}$ are used here as model systems. $\mathrm{Ga}_{x} \mathrm{In}_{1-x} \mathrm{P}$ was chosen due to the large difference in covalent radius between $\mathrm{In}$ and $\mathrm{Ga}$. $\mathrm{C}_{\mathrm{IV}}^{0}$ in $\mathrm{Si}_{x} \mathrm{Ge}_{1-x}$ was chosen since the isovalency of the atoms minimizes the ionic contributions to the bonds and the neutral charge state of the dopant minimizes the electrostatic contributions to the formation energy. We first consider disordered alloys, then the CuPt-ordered [22] phase of the alloys, and finally we study the controlled environment of thin multilayers. All systems are relaxed fully and their lattice constants all agree with the linear interpolation of the LDA values to an error of less than $0.05 \%$. 
The defect formation energy is calculated as

$$
\varepsilon^{\mathrm{form}}(q)=E_{\mathrm{def}}^{\mathrm{Tot}}(q)-E_{\text {ideal }}^{\mathrm{Tot}}-\sum_{i} n_{i} \mu_{i}+q\left(\varepsilon_{F}+\varepsilon_{V}\right),
$$

where $E_{\text {def }}^{\text {Tot }}(q)$ and $E_{\text {ideal }}^{\text {Tot }}$ are the total energies of the supercell with and without the defect in charge state $q . n_{i}$ is the number of atoms of type $i$ and chemical potential $\mu_{i}$ added in order to form the defect. $\varepsilon_{V}$ is the energy of the valence band maximum and $\varepsilon_{F}$ is the Fermi level. Since strongly $p$-type materials are considered the Fermi level is approximated to be located at the valence band maximum. Stoichiometric values are used for the chemical potentials.

Comparing the formation energies of dopants in $\mathrm{Si}_{0.5} \mathrm{Ge}_{0.5}$ and CuPt-ordered $\mathrm{Ga}_{0.5} \mathrm{In}_{0.5} \mathrm{P}$ to those of the pure materials, our calculations show that the formation energy of $\mathrm{Zn}_{\text {In }}^{-}$is $0.35 \mathrm{eV}$ lower in $\mathrm{Ga}_{0.5} \mathrm{In}_{0.5} \mathrm{P}$ than in pure $\mathrm{InP}$, whereas that of $\mathrm{Zn}_{\mathrm{Ga}}^{-}$is relatively unchanged, $0.01 \mathrm{eV}$ higher than in pure GaP. The corresponding energies in the disordered material are 0.27 and $-0.07 \mathrm{eV}$ on average. For cadmium, it is instead $\mathrm{Cd}_{\mathrm{Ga}}^{-}$that changes the most since its formation energy is $0.38 \mathrm{eV}$ lower in $\mathrm{Ga}_{0.5} \mathrm{In}_{0.5} \mathrm{P}$ than in pure $\mathrm{GaP}$, while the change for $\mathrm{Cd}_{\mathrm{In}}^{-}$is insignificant, $0.07 \mathrm{eV}$ relative to pure InP. Since $\mathrm{Zn}$ and $\mathrm{Cd}$ are isovalent their most significant difference is size and therefore our results suggest that the formation energy depends on the local structure and especially the local strain of the material. It is noteworthy that the formation energy is not a unique function of $x$ and unlike properties such as the band gap or the lattice constant, it does not follow a single monotonic relation.

To better to understand how the local environment affects the formation energy of dopants, we study the same dopants as discussed above, but in a multilayer geometry: $\mathrm{GaP}_{x} / \mathrm{InP}_{1-x}$ with $x=0.33,0.5$, and 0.67 , and $\mathrm{C}_{\mathrm{IV}}^{0}$ in $\mathrm{Si}_{0.5} / \mathrm{Ge}_{0.5}$. Allowing both the ions and the volume of the multilayer supercells to relax fully, it is found that there are tetragonal distortions of the layers. This distortion is such that there is a lattice matching at the interface and to preserve the unit cell volume the smaller $\mathrm{GaP}(\mathrm{Si})$ unit cell is shortened in the direction perpendicular to the interface while the larger unit cell of $\operatorname{InP}(\mathrm{Ge})$ is elongated in the same direction. Values of the lattice constants parallel to the interface $\left(a_{\|}\right)$and perpendicular to it $\left(a_{\perp}^{i}\right)$ are tabulated in Table I. Further, it is found that the bond distances between two given species are constant to within $0.05 \AA$. For a given $x$ all multilayer structures considered are therefore found to be highly uniform within the layers of each material type, i.e., $a_{\perp}^{i}$ does not depend on position in the multilayer, although there naturally are discontinuities at the interfaces. As a result, the formation energy of a dopant is the same inside a layer (to within $0.06 \mathrm{eV}$ ), independent of the distance to the interfaces.

In Fig. 1(a) the dependence of the formation energy of $\mathrm{C}_{\mathrm{IV}}^{0}$ on the lattice constant is shown for pure $\mathrm{Si}$ and $\mathrm{Ge}$
TABLE I. Lattice constants of multilayer structures $(\AA)$. The local average lattice constant is defined as $a_{\text {loc. }}^{i}=\left(a_{\|}^{2} a_{\perp}^{i}\right)^{1 / 3}$, and the overall average as $a_{\mathrm{av} .}=x a_{\mathrm{loc}}^{A}+(1-x) a_{\mathrm{loc}}^{B}$. To the given accuracy, the linearly interpolated values, as predicted by Vegard's law, coincide with $a_{\text {av. }}$ and are therefore omitted.

\begin{tabular}{lcccccc}
\hline \hline$x$ & $a_{\|}$ & $a_{\perp}^{\mathrm{Si}}$ & $a_{\perp}^{\mathrm{Ge}}$ & $a_{\text {av. }}$ & $a_{\text {loc. }}^{\mathrm{Si}}$ & $a_{\text {loc. }}^{\mathrm{Ge}}$ \\
\hline 0.5 & 5.50 & 5.30 & 5.71 & 5.50 & 5.43 & 5.57 \\
\hline \hline \hline$x$ & $a_{\|}$ & $a_{\perp}^{\mathrm{GaP}}$ & $a_{\perp}^{\mathrm{InP}}$ & $a_{\text {av. }}$ & $a_{\text {loc. }}^{\mathrm{GaP}}$ & $a_{\text {loc. }}^{\mathrm{InP}}$ \\
\hline 0.33 & 5.68 & 5.10 & 5.97 & 5.68 & 5.49 & 5.78 \\
0.5 & 5.61 & 5.17 & 6.05 & 5.61 & 5.46 & 5.76 \\
0.67 & 5.54 & 5.24 & 6.12 & 5.54 & 5.44 & 5.73 \\
\hline \hline
\end{tabular}

(dotted and full lines) [23]. The lattice constant is varied from the LDA equilibrium lattice constant of $\mathrm{Si}$ to that of Ge. The same thing is shown for $\mathrm{Zn}_{\text {III }}^{-}$and $\mathrm{Cd}_{\text {III }}^{-}$in $\mathrm{GaP}$ and InP in Fig. 1(b). Analyzing the slope of the curves it is seen that a dopant of equal size as the substituted host atom has

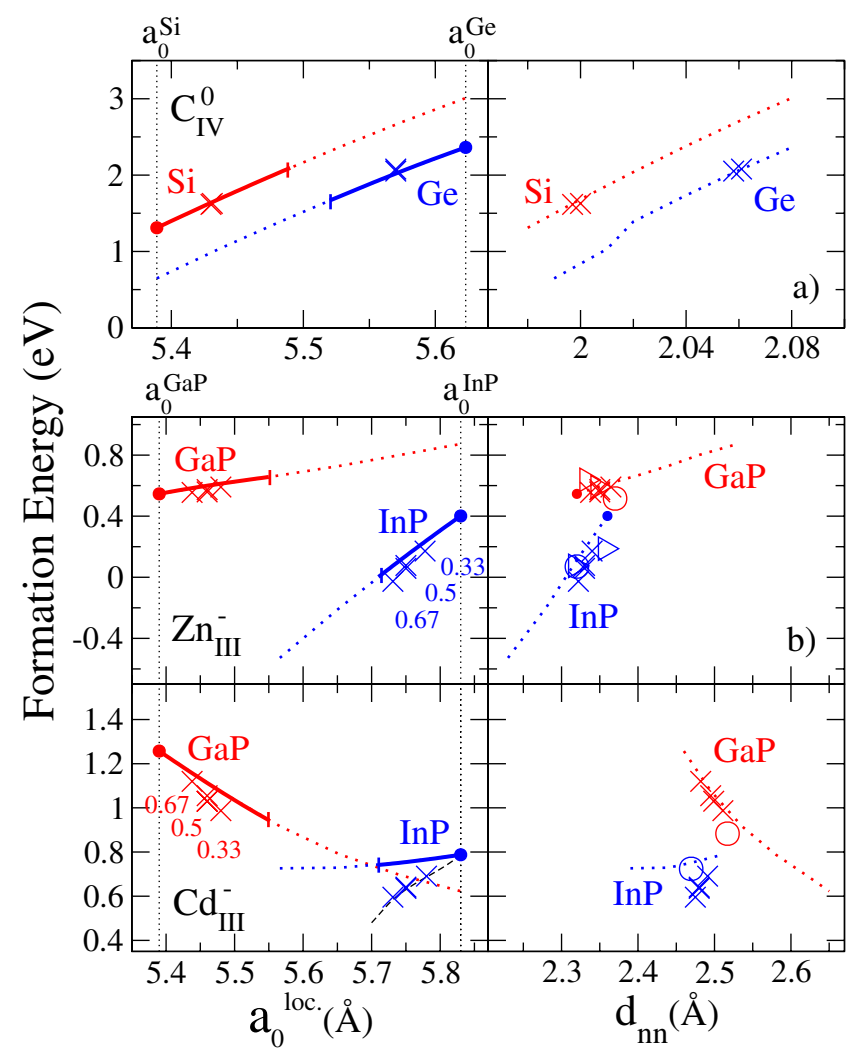

FIG. 1 (color online). Correlation of formation energy of $\mathrm{C}_{\mathrm{IV}}^{0}$, $\mathrm{Zn}_{\mathrm{III}}^{-}$, and $\mathrm{Cd}_{\mathrm{III}}^{-}$and the local lattice constant (left) or the average bond distance (right). Dotted curves show the formation energy dependence of the pure materials. Following the analytical treatment of Ref. [28] the local lattice constants of the multilayers are calculated in the entire range $x=0 \rightarrow 1$ and the ranges they span are indicated by full lines. Values from multilayers, ordered compounds and disordered alloys are denoted by $\times, \bigcirc$, and $\triangleright$, respectively. 
little dependence on strain since the equilibrium bond distances of the dopant to the host structure are close to those of the unperturbed host. For a smaller sized dopant, the formation energy will decrease upon compression and the dependence on the lattice constant has a positive slope. Following the same argumentation, the strain dependence of dopant atoms larger than the host has a negative slope.

For multilayers one needs to use the local lattice constant (Table I) as the relevant parameter. It is defined as $a_{\text {loc. }}^{i}=\left(a_{\|}^{2} a_{\perp}^{i}\right)^{1 / 3}$. The formation energy of the $\mathrm{Si}_{x} / \mathrm{Ge}_{1-x}$ multilayer lies very close to the data for the externally strained materials (dotted lines). The formation energies shown in Fig. 1(b) for the $\mathrm{GaP}_{x} / \mathrm{InP}_{1-x}$ multilayers also show close resemblance to the values given by the externally strained $\mathrm{GaP}$ and InP materials. For alloys and compounds it is not possible to define a local lattice constant and instead the average bonding distance is used as the gauge of the lattice $\left(d_{\mathrm{nn}}\right)$. It is clear that also for these systems the formation energy lies close to the values of the externally strained materials [24]. Hence, the data in Fig. 1 illustrates that it is the relative size of the dopant atom which is the most important parameter that governs the formation energy.

Following the size argument above, the formation energy dependence on strain of the smaller $C$ dopant has a positive slope in both $\mathrm{Si}$ and $\mathrm{Ge}$. Since the local lattice constant in the $\mathrm{Si}(\mathrm{Ge})$ layers of multilayer $\mathrm{Si}_{0.5} \mathrm{Ge}_{0.5}$ is $5.43 \AA(5.47 \AA)$ as compared to $5.39 \AA(5.62 \AA)$ in pure $\mathrm{Si}$ $(\mathrm{Ge}), \mathrm{C}_{\mathrm{Si}}^{0}\left(\mathrm{C}_{\mathrm{Ge}}^{0}\right)$ is expected to have a lower (higher) equilibrium concentration in multilayer $\mathrm{Si}_{0.5} \mathrm{Ge}_{0.5}$ than in pure $\mathrm{Si}(\mathrm{Ge}) . \mathrm{Cd}$ is of equal size to In, therefore the formation energy of $\mathrm{Cd}_{\text {III }}^{-}$is expected to have a weak dependence on the lattice constant in the InP multilayer. Further, it is expected to cause a strain in the surrounding structure when substituting a smaller Ga atom, which gives the lattice dependence curve a negative slope in the GaP multilayer, as seen in Fig. 1(b). $\mathrm{Zn}$ is instead of equal size to $\mathrm{Ga}$, which results in a flat dependence of the formation energy on the lattice constant for $\mathrm{Zn}_{\mathrm{Ga}}^{-}$and therefore the solubility on Ga sites is not expected to change significantly with the $x$. The formation energy of $\mathrm{Zn}_{\text {In }}^{-}$, on the other hand, has a strong dependence on the lattice constant. For pure InP, $x=0$, the formation energy of $\mathrm{Zn}_{\mathrm{In}}^{-}$is $0.39 \mathrm{eV}$ but it is reduced toward zero as $x$ approaches 1 . Since $\mathrm{Zn}$ will be incorporated on the sites where it has the lowest formation energy, this means that the equilibrium solubility of $\mathrm{Zn}$ in $\mathrm{Ga}_{x} \mathrm{In}_{1-x} \mathrm{P}$ can be increased by several orders of magnitude by reducing the In content and raising $x$. Unlike the other dopants studied here, $\mathrm{Zn}$ shows that the solubility can be considerably higher in $\mathrm{Ga}_{x} \mathrm{In}_{1-x} \mathrm{P}$ than in either $\mathrm{GaP}$ or $\mathrm{InP}$.

From the results for disordered GaInP it is clear that the sublattice in which substitution takes place is of main importance; the formation energy of $\mathrm{Zn}_{\text {In }}$ will follow the trend of externally strained InP independent of the nearestneighbor environment. That the type of atom substituted is important shows that the size argument is synonymous to that of strain relief; if compressive (expansive) strain is relieved by the substitution with a smaller (larger) dopant it will result in a lower formation energy relative to the unstrained material. As for the internal strain, the disordered alloy will have residual strain in much the same way as multilayer structures. This means that if the local lattice constants are known, either from experiment or through analytical calculation, the formation energy of the dopant can be calculated for the pure materials at those local lattice constants to provide an estimate $( \pm 0.1 \mathrm{eV})$ of the formation energies in the alloy or multilayer. This provides a means of determining how alloying will influence the dopant concentration and thereby the charge carrier concentration.

Several experimental findings can be explained by this size argument: Hong et al. have found that the equilibrium solubility of $\mathrm{Sb}$ in $\mathrm{Ge}_{0.1} \mathrm{Si}_{0.9}$ decreases with compressive strain, which can be explained by Sb being larger than both $\mathrm{Si}$ and $\mathrm{Ge}$ [25]. The equilibrium solubility of $\mathrm{Si}$ in GaAs, on the other hand, would be expected to decrease under expansive strain due to the smaller size of the $\mathrm{Si}$ atom. In accordance, it has been observed that the Si concentration, both in the Ga and As sublattice, decreases with the incorporation of In, which increases the lattice constant of $\mathrm{Ga}_{1-x} \mathrm{In}_{x} \mathrm{As}$ [26]. The measured decrease in charge carrier concentration of $\mathrm{Ga}_{0.8} \mathrm{In}_{0.2} \mathrm{Sb}$, as compared to $\mathrm{GaSb}$, can also be explained by the decreasing solubility of $\mathrm{Si}_{\mathrm{Sb}}$ with increasing In content [27].

To generalize, the equilibrium solubility of a dopant in a material can be raised by strategic alloying as follows: if the dopant atoms are smaller (larger) in size than the host atoms $A$, one should alloy with a material $B$ which has a lattice constant $a_{0}^{A}>a_{0}^{B}\left(a_{0}^{A}<a_{0}^{B}\right)$ and a formation energy larger than that of material $A$, i.e. $\varepsilon_{A}^{\text {form }}<\varepsilon_{B}^{\text {form }}$ (so that the dopants sit on $A$ sites). That is, a substitution that leads to strain relief will be energetically favorable and lead to an increasing equilibrium solubility of that dopant.

Turning back to Eq. (1), it is seen that the equilibrium concentration is not solely determined by the formation energy; changing $x$ will also change the number of possible lattice sites $N_{s}$. Taking the example of thin multilayers, we can rewrite Eq. (1) as a function of $x$ which allows us to find the value of $x$ that maximizes the concentration. For the case of $\mathrm{Zn}_{\text {In }}$ in $\mathrm{GaP}_{x} / \mathrm{InP}_{1-x}$, the number of In lattice sites is simply $N_{\text {InP }}=(1-x) N_{\text {tot }}$. By repeatedly minimizing the total energy for varying multilayer thicknesses according to Ref. [28], we can find $a_{0}^{\text {loc. }}$ as a function of $x$ (for consistency LDA values of $a_{0}^{\mathrm{GaP}}$ and $a_{0}^{\mathrm{InP}}$ are used). Combining this with the formation energy's dependence on the local lattice constant of Fig. 1 finally gives the formation energy as a function of $x$. The concentration in Eq. (1) can then be expressed entirely as a function of $x$. The resulting concentration of $\mathrm{Zn}_{\text {In }}^{-}$in $\mathrm{GaP}_{x} / \mathrm{InP}_{1-x}$ is plotted in Fig. 2 and it is seen that the maximum solubility is 


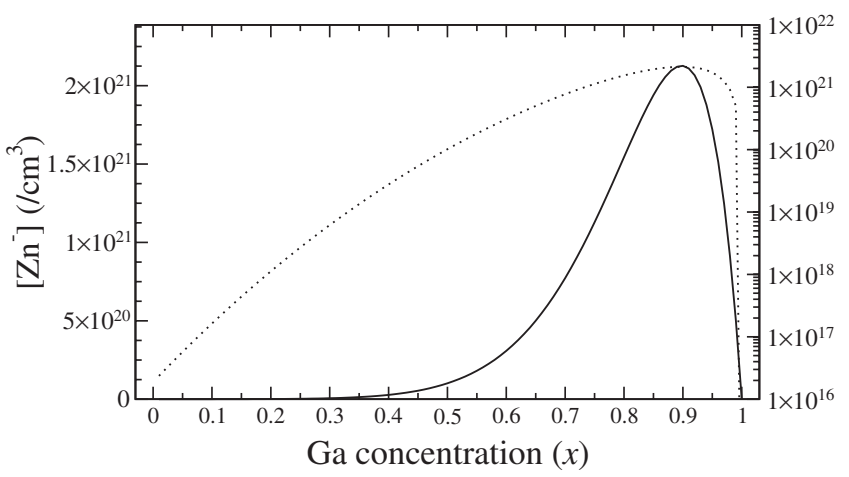

FIG. 2. The $\mathrm{Zn}_{\text {In }}^{-}$concentration in multilayered $\mathrm{GaP} / \mathrm{InP}$ as a function of $x$. The dotted curve corresponds to right $y$ axis. The relative increase of 5 orders of magnitude is reliable, although absolute values may not be due to the sensitivity to residual errors and neglect of formation entropy contributions.

achieved for $x=0.9$. Although this analysis is carried out for a $\mathrm{GaP}_{x} / \mathrm{InP}_{1-x}$ multilayer, the key mechanism of the formation energy's dependence on the local strain is general and therefore the same trend should be valid for all $\mathrm{Ga}_{x} \mathrm{In}_{1-x} \mathrm{P}$ structures. Figure 2 suggests that an increase of the dopant concentration can be achieved by strategic alloying of the host material or formation of multilayers, and that the doping concentration can be made to increase by 5 orders of magnitude.

To conclude, we have shown that the formation energy of dopants can have a strong dependence on the local lattice constant, depending on the size mismatch of the dopant and the host atom. The formation energies of dopants in alloys and multilayers are found to correlate well with those of the externally strained materials at the corresponding lattice constant. Therefore the dopant solubility can be predicted in an alloy or multilayer if only the dependence on strain and the local lattice constant are known. Furthermore, it has been shown that the equilibrium solubility of a dopant can be significantly increased by tuning the local lattice constant through strategic alloying and that it can exceed those of the pure materials by several orders of magnitude. For $\mathrm{Zn}_{\text {III }}^{-}$in $\mathrm{Ga}_{x} \mathrm{In}_{1-x} \mathrm{P}$ it has been shown that the maximal equilibrium solubility is achieved for $x=0.9$, at which point it is increased by 5 orders of magnitude.

[1] A. Vailionis et al., Phys. Rev. Lett. 82, 4464 (1999).

[2] G. Glass et al., Phys. Rev. B 61, 7628 (2000).

[3] H. Kim et al., J. Appl. Phys. 89, 194 (2001).
[4] M. Weyers and M. Sato, Appl. Phys. Lett. 62, 1396 (1993).

[5] R. A. Hamm et al., Appl. Phys. Lett. 54, 2586 (1989).

[6] C. R. Abernathy et al., Appl. Phys. Lett. 55, 1750 (1989).

[7] E. A. Ekimov et al., Nature (London) 428, 542 (2004) and references therein.

[8] B. E. Weir et al., Appl. Phys. Lett. 65, 737 (1994).

[9] J. Falta et al., Appl. Surf. Sci. 123/124, 538 (1998).

[10] H.-J Gossmann, F. C. Unterwald, and H.S. Luftman, J. Appl. Phys. 73, 8237 (1993).

[11] S. B. Zhang and Su-H. Wei, Phys. Rev. Lett. 86, 1789 (2001).

[12] X. Luo, S. B. Zhang, and S. H. Wei, Phys. Rev. Lett. 90, 026103 (2003).

[13] X. Luo, S. B. Zhang, and S.H. Wei, Phys. Rev. B 70, 033308 (2004).

[14] L. Romano et al., Phys. Rev. Lett. 97, 136605 (2006); N. Chen et al., Phys. Rev. B 54, 8516 (1996); Q. X. Zhao et al., J. Appl. Phys. 97, 073714 (2005); D. T. Neilson et al., Appl. Phys. Lett. 70, 2031 (1997); B.-D. Liu et al., J. Appl. Phys. 72, 2767 (1992); F. Brunet et al., J. Appl. Phys. 83, 181 (1998).

[15] S. Chaudry and M. E. Law, J. Appl. Phys. 82, 1138 (1997); G. Charitat and A. Martinez, J. Appl. Phys. 55, 909 (1984); A. Karoui et al., J. Appl. Phys. 96, 3255 (2004); N. S. Bennett et al., Appl. Phys. Lett. 89, 182122 (2006).

[16] B. Sadigh et al., Appl. Phys. Lett. 80, 4738 (2002).

[17] N. S. Rytova and E. V. Soloveva, Sov. Phys. Semicond. 20, 869 (1986); K. Tanahashi et al., Physica (Amsterdam) 273B, 493 (1999).

[18] D. B. Laks et al., Phys. Rev. Lett. 66, 648 (1991); S. B. Zhang and J.E. Northrup, Phys. Rev. Lett. 67, 2339 (1991).

[19] W. Kohn and L. J. Sham, Phys. Rev. 140, A1133 (1965).

[20] D. Vanderbilt, Phys. Rev. B 41, 7892 (1990); G. Kresse and J. Hafner, J. Phys. Condens. Matter 6, 8245 (1994).

[21] A. Höglund, C. W. M. Castleton, and S. Mirbt, Phys. Rev. B 72, 195213 (2005) and references therein; A. Höglund, C. W. M. Castleton, and S. Mirbt (to be published).

[22] A. Gomyo, T. Suzuki, and S. Iijima, Phys. Rev. Lett. 60, 2645 (1988); M. Kondow et al., Phys. Rev. Lett. 63, 884 (1989).

[23] $\mu_{i}$ and $\varepsilon_{V}$ of Eq. (2) must be treated as functions of $x$.

[24] The discrepancy for $\mathrm{Cd}_{\text {In }}^{-}$is due to the difference in $\mu_{\text {In }}$ between InP and $\mathrm{GaP}_{x} / \mathrm{InP}_{1-x}$. Correcting this by using the multilayer $\mu_{\text {In }}$ for the pure InP gives the dashed black line in Fig. 1(b).

[25] S. Q. Hong, Q. Z. Hong, and J. W. Mayer, Appl. Phys. Lett. 63, 2053 (1993).

[26] V. A. Joshkin et al., Mater. Sci. Eng. B 26, 7 (1994).

[27] H. Ehsani et al., Appl. Phys. Lett. 69, 3863 (1996).

[28] A. Broddefalk et al., J. Magn. Magn. Mater. 241, 260 (2002). 